\title{
Interplay of supersaturation and solubilization: Lack of correlation between concentration-based supersaturation measurements and membrane transport rates in simulated and aspirated human fluids
}

\author{
Ahmed Elkhabaz ${ }^{\dagger}$, Dana E. Moseson ${ }^{\dagger}$, Joachim Brouwers ${ }^{\ddagger}$, Patrick Augustijns ${ }^{\ddagger}$ and Lynne S. Taylor ${ }^{\dagger *}$. \\ 'Department of Industrial and Physical Pharmacy, College of Pharmacy, Purdue University, West \\ Lafayette, Indiana, USA \\ †Drug Delivery and Disposition, KU Leuven, Leuven, Belgium
}

*Corresponding author. 1staylor@purdue.edu 


\begin{abstract}
Supersaturating formulations are increasingly being used to improve the absorption of orally administered poorly water-soluble drugs. To better predict outcomes in vivo, we must be able to accurately determine the degree of supersaturation in complex media designed to provide a surrogate for the gastrointestinal environment. Herein, we demonstrate that relying on measurements based on consideration of the total dissolved concentration, leads to under-estimation of supersaturation and consequently membrane transport rates. Crystalline and amorphous solubilities of two compounds, atazanavir and posaconazole, were evaluated in six different media. Concurrently, diffusive flux measurements were performed in a side-byside diffusion cell to determine the activity-based supersaturation by evaluating membrane transport rates at the crystalline and amorphous solubilities. Solubility values were found to vary in each medium due to different solubilization capacities. Concentration-based supersaturation ratios were also found to vary for the different media. Activity-based measurements however, were largely independent of the medium, leading to relatively constant values for the estimated supersaturation. These findings have important consequences for modeling and prediction of supersaturation impact on the absorption rate, as well as for better defining the thermodynamic driving force for crystallization in complex media.
\end{abstract}

Keywords. Biorelevant media; supersaturation; membrane transport; amorphous; crystalline. 


\section{Introduction}

Modern drug discovery strategies have led to an increase in therapeutic candidates with complex molecular structures and challenging physicochemical properties. ${ }^{1,2}$ One major issue with these prospective drugs is their poor aqueous solubility which potentially hinders oral delivery. As a result, there has been a great deal of interest in formulations that generate supersaturated solutions of these practically insoluble compounds. ${ }^{3,4}$ Supersaturation is attained when the thermodynamic activity (and the related entity, the chemical potential) of a solute in the solution phase is higher than that of the solute at equilibrium with the most stable crystalline form. ${ }^{5}$ Supersaturation is typically reported as the supersaturation ratio $\left(S R_{\text {conc }}\right)$ using concentration measurements:

$S R_{\text {conc }}=\frac{C}{C_{e q}}(1)$

where $C$ is the concentration of the drug in the medium of interest and $C_{e q}$ is the drug crystalline solubility in exactly the same medium (i.e. identical $\mathrm{pH}$, buffer components, ionic strength, micellar species etc). From a clinical perspective, the presence of a drug at a higher concentration than its equilibrium solubility i.e. in a supersaturated state, provides an enhanced driving force for absorption, which in turn can positively impact bioavailability. ${ }^{6-11}$ Hence, diffusive flux across a membrane increases linearly with supersaturation, up to a certain supersaturation limit. ${ }^{10,12}$ Beyond this limit, liquid-liquid phase separation (LLPS) occurs, with the formation of colloidal drug-rich aggregates. ${ }^{12,13}$ The occurrence of LLPS marks the upper limit of supersaturation which can be achieved, whereby the concentration at which LLPS occurs is closely related to the amorphous solubility of a compound. Since no further increase in the free drug concentration in the solution phase can be achieved above the amorphous solubility, the diffusive flux becomes relatively constant for concentrations beyond that limit. ${ }^{10}$ Nevertheless, the formation of these colloidal species has been hypothesized to be beneficial for oral drug delivery, by serving as a reservoir and continuously replenishing the absorbed drug. ${ }^{10,14}$ Diffusion through the unstirred water layer is often considered the ratelimiting step for intestinal uptake of highly permeable lipophilic compounds. ${ }^{15-17}$ Therefore, particle drifting into the unstirred water layer, has also been suggested as a mechanism by which absorption is enhanced by colloidal drug aggregates. ${ }^{18,19}$ It should be noted that supersaturated solutions as well as phase separated systems containing colloidal drug-rich aggregates will only exist if crystallization is prevented or retarded.

When assessing supersaturating formulations during preclinical development, it is important to consider a variety of factors including the physicochemical properties of the drug and the relative stability of the supersaturated state in the dissolution medium. Understanding supersaturation thermodynamics for relevant in vivo conditions can be advantageous from a formulation design standpoint. Thus, performing 
supersaturation assessment in biorelevant conditions is pivotal for reasonable prediction of eventual intraluminal performance. Biorelevant media have gained increasing attention in pharmaceutical development, where their utilization is intended to better mimic the composition of the gastrointestinal fluids, as compared to simple aqueous buffer solutions. The presence of solubilizing species including bile salts and phospholipids, in addition to other solution properties such as $\mathrm{pH}$, can greatly alter supersaturation and phase behavior of lipophilic compounds. In particular, for supersaturating formulations, the impact of dissolution media on factors such as the amorphous solubility (maximum degree of supersaturation) and supersaturation duration, is poorly understood. Further, comparisons between the thermodynamic properties and membrane transport rates of supersaturated drug solutions in simulated versus human intestinal fluids have not been made, yet are essential to enhance the predictability of in vitro assessments. Recent characterization studies of human intestinal fluids (HIF) have identified six main bile salts that constitute approximately $98.4 \%$ of the biological surfactant content in the gastrointestinal tract. ${ }^{20}$ These include sodium taurocholate (STC), sodium taurodeoxycholate (STDC), sodium taurochenodeoxycholate (STCDC), sodium glycocholate (SGC), sodium glycodeoxycholate (SGDC), and sodium glycochenodeoxycholate (SGCDC). The molecular structure of bile salts is distinctive with a steroid ring system to which three (STC and SGC) or two hydroxyl groups (STDC, STCDC, SGDC and SGCDC) are attached. ${ }^{21-23}$ The critical micelle concentration (CMC) of bile salts is usually not well-defined, since they exhibit a complex pattern of self-association, including dimerization as well as the formation of micellar aggregates and higher oligomers/multimers. ${ }^{24-26}$ Variations in the substitution/orientation of the hydroxyl or presence of tauro/glyco groups on the bile salt structure alter the CMC range, resulting in different solubilization capacities among the various bile salts. ${ }^{27}$ In addition to bile salts, lecithin (composed of a mixture of phospholipids) is another main component of HIF. The presence of lecithin lowers the CMC of bile salts and leads to the formation of mixed micelles. ${ }^{28,29}$

Current commercial biorelevant media such as fasted/fed-state simulated intestinal fluid (Fa/FeSSIF) contain a single bile salt, STC. The first version of FaSSIF (FaSSIF-V1) utilizes a sodium phosphate buffer and contains STC and lecithin in concentrations of $3 \mathrm{mM}$ and $0.75 \mathrm{mM}$, respectively. ${ }^{30}$ The refined version of FaSSIF (FaSSIF-V2), introduced in $2008,{ }^{31}$ replaces the sodium phosphate buffer with a maleic acidbased buffer, and decreases the lecithin content to $0.2 \mathrm{mM}$. Both versions of FaSSIF have a pH of 6.5. However, both commercial media are oversimplified in their composition relative to HIF since they contain only a single bile salt (STC). Studies from our group have shown that bile salts are not interchangeable in terms of their impact on the solution thermodynamic properties, ${ }^{32}$ or on the stability of supersaturated solutions to crystallization. ${ }^{33,34}$ Moreover, little is known about the behavior of supersaturated drug solutions in aspirated HIF, and how well this behavior can be mimicked by simulated biorelevant media. The difference in the composition of the commercially-available biorelevant media versus HIF could affect 
their relative solubilization capacity, both for crystalline drug, as well as for supersaturated solutions. Theoretically, differences in solubilization capacity between media may impact the perceived supersaturation extent when evaluated based on the total dissolved concentration (Equation 1). ${ }^{35,36}$ This could be particularly important when evaluating aspirated fluids following oral dosing to assess the extent to which supersaturation has occurred, as well as using simulated media to predict in vivo supersaturation. Thus, it is imperative to evaluate supersaturation based on measurement of the solute activity, which can be viewed as the 'effective' drug concentration, and to determine how well the concentration-based value correlates with this more rigorous parameter.

In this work, the hypothesis to be tested is that the maximum supersaturation that can be achieved is dictated by the effective drug concentration (i.e. the solute activity), at the amorphous solubility of the compound. Further, the effective drug concentration in the presence of an amorphous precipitate is expected to be largely independent of the type of biorelevant medium employed. We further hypothesize that using total dissolved concentrations to determine the supersaturation ratio, as per Equation 1, will lead to incorrect conclusions about the thermodynamic driving force for membrane transport and solute crystallization. To test this hypothesis, we assess the impact of different media on the crystalline and amorphous solubilities, supersaturation ratios and membrane transport rates, for two poorly-soluble drugs. Here, the assumption is that the membrane transport rate is dictated by the solute activity gradient. ${ }^{37}$ The two model compounds used are atazanavir ( $\log$ P: 4.5, pKa: 4.52) ${ }^{38}$ and posaconazole (log P: 4.6, pKa: 3.6 and 4.6). ${ }^{39,40}$ These compounds are both weak bases, and therefore likely to undergo supersaturation upon transfer from the gastric environment to the small intestine. The media used included two simple buffers namely sodium phosphate and maleic acid buffer, and two commercial biorelevant media, FaSSIF-V1 and FaSSIF-V2. Additionally, fasted-state aspirated human intestinal fluid (FaHIF) was used to systematically evaluate differences between both simulated media and aspirated biologic fluid. Finally, a modified version of FaSSIF comprised of the six most prevalent bile salts, plus lecithin, (Composite-FaSSIF) was prepared to more closely mimic the composition of FaHIF, from a simulated medium perspective. By measuring the crystalline solubility, the amorphous solubility and diffusive flux across a membrane, the varying impact of all six media on the thermodynamic properties of supersaturated solutions for two compounds was investigated.

\section{Materials}

Atazanavir (free base) and posaconazole (Form I), shown in Figure 1, were purchased from ChemShuttle (Hayward, CA). Hydroxypropyl methylcellulose acetyl succinate (HPMC-AS) MF grade was supplied by Shin-Etsu Chemical Co. (Tokyo, Japan). Acetonitrile, dichloromethane (DCM), dimethyl sulfoxide (DMSO), hydrochloric acid $(\mathrm{HCl})$, maleic acid, methanol, sodium chloride, and sodium phosphate 
monobasic monohydrate were bought from Fisher Scientific (Pittsburgh, PA). Sodium hydroxide (NaOH) was purchased from Avantor Performance Materials, LLC (Radnor, PA). A regenerated cellulose membrane with a molecular weight cutoff (MWCO) of 6-8 kDa was acquired from Spectrum Laboratories, Inc. (Rancho Dominguez, CA). FaSSIF/FeSSIF/FaSSGF and FaSSIF-V2 powders were purchased from Biorelevant (London, UK). L- $\alpha$-Phosphatidylcholine (lecithin, $\geq 99 \%$ ), sodium taurocholate hydrate, (STC $[\geq 97 \%]$ ), sodium glycocholate, (SGC $[\geq 95 \%]$ ), sodium taurodeoxycholate, (STDC $[\geq 95 \%]$ ), sodium glycodeoxycholate, (SGDC [ $\geq 97 \%]$ ), sodium taurochenodeoxycholate, (STCDC [ $\geq 97 \%]$ ), and sodium glychochenodeoxycholate, (SGCDC [ $\geq 97 \%]$ ) were obtained from Sigma-Aldrich (St. Louis, MO). Fastedstated human intestinal fluid (FaHIF) was collected from healthy human volunteers as detailed below.
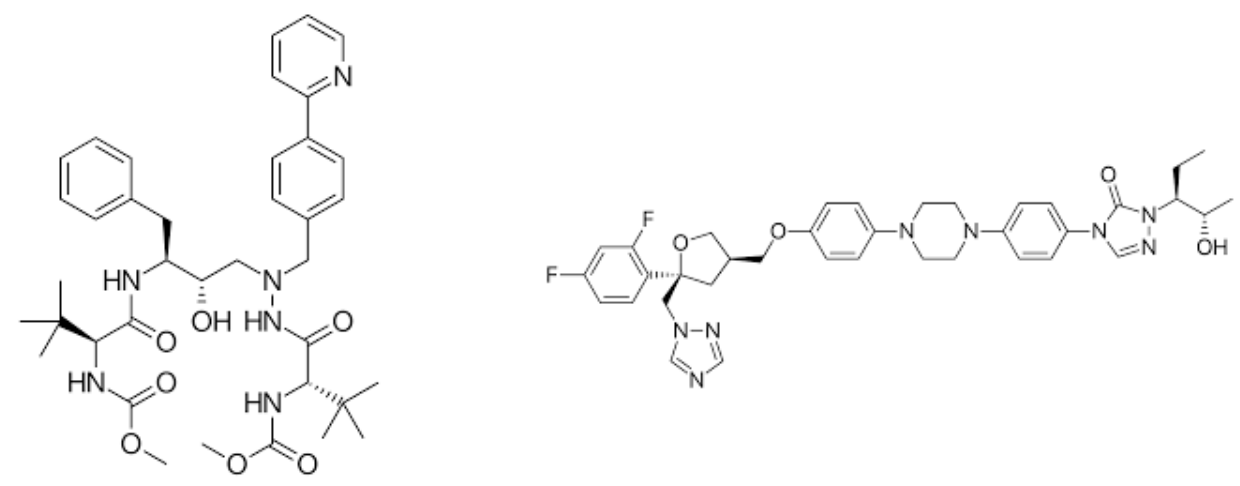

Figure 1. Molecular structures of atazanavir (left), and posaconazole (right).

\section{Methods}

\section{Media selection and preparation}

Six different media were used in this study. Two blank (without bile salts/lecithin) aqueous buffers were prepared as described previously. ${ }^{30,31}$ These two buffers are referred to as FaSSIF-V1 blank phosphate buffer (buffer-V1) and FaSSIF-V2 blank maleic acid buffer (buffer-V2), respectively. FaSSIF/FeSSIF/FaSSGF powder was added to buffer-V1 to create FaSSIF-V1, ${ }^{30}$ whilst FaSSIF-V2 powder was added to buffer-V2 to make FaSSIF-V2. ${ }^{31}$ Composite-FaSSIF was prepared using buffer-V1. The composition of composite-FaSSIF was based on a recent characterization of FaHIF by Riethorst et al., ${ }^{20}$ where the total bile salts concentration was $3 \mathrm{mM}$, which is equivalent to the STC concentration in FaSSIFV1 and FaSSIF-V2. In addition, $0.2 \mathrm{mM}$ of lecithin was added to this medium. Preparation of compositeFaSSIF was performed as described before. ${ }^{36}$ Briefly, all six bile salts were weighed, added to buffer-V1, stirred and then allowed to settle for $30 \mathrm{~min}$. Meanwhile, $0.2 \mathrm{mM}$ of lecithin was dissolved in dichloromethane (DCM) and titrated into the aqueous bile salt mixture. The composite-FaSSIF mixture was 
then stirred at $500 \mathrm{rpm}$, maintained at $50^{\circ} \mathrm{C}$ for $30 \mathrm{~min}$ to evaporate the organic phase from the aqueous mixture. The initial volume of added DCM was always kept to less than $1 \%$ of the total solution volume. All of the aforementioned media were adjusted to $\mathrm{pH}$ 6.5. The composition of the various media is summarized in Table 1.

Table 1. Detailed composition of the bile salts and lecithin for FaSSIF-V1, FaSSIF-V2 and composite-FaSSIF.

FaSSIF-V1

STC

STDC

STCDC

SGC

SGDC

SGCDC

Lecithin
$3 \mathrm{mM}$

$0.75 \mathrm{mM}$
FaSSIF-V2

$3 \mathrm{mM}$

$0.36 \mathrm{mM}$

$0.36 \mathrm{mM}$

$0.18 \mathrm{mM}$

$0.84 \mathrm{mM}$

$0.45 \mathrm{mM}$

$0.81 \mathrm{mM}$

$0.2 \mathrm{mM}$

\section{Sampling and handling of FaHIF}

Human intestinal fluids were collected at the University Hospitals Leuven as part of a previously published study. ${ }^{20}$ The sampling procedure was approved by the Ethics Committee Research UZ/KU Leuven (reference numbers ML7918 and S53791); healthy Caucasian volunteers were enrolled in the study after giving written informed consent. Briefly, intestinal fluids were collected from the duodenum (near the ligament of Treitz) from 20 healthy volunteers every ten minutes for a period of $90 \mathrm{~min}$ in both fasted and fed state. After an overnight fast of at least $12 \mathrm{~h}$ (no food and only water), volunteers were given $250 \mathrm{~mL}$ of water before initiating the sampling of fasted state intestinal fluids. Following fasted state sampling, 400 $\mathrm{mL}$ of Ensure ${ }^{\circledR}$ Plus (Abbott Nutrition, Zwolle, the Netherlands) was ingested to simulate a standard meal. Fed state sampling was initiated after drinking $250 \mathrm{~mL}$ water, $20 \mathrm{~min}$ after the intake of the liquid meal. For each volunteer, the collected fractions were pooled to obtain a fasted and fed state individual volunteer pool. For the present study, a mixed pool of FaHIF was created by gently mixing samples from 16 individual volunteer pools. Afterwards, these samples were kept frozen at $-80^{\circ} \mathrm{C}$ until use. The $\mathrm{pH}$ of the FaHIF pool used in this study was measured using a Mettler Toledo FiveEasy ${ }^{\mathrm{TM}}$ FE20 benchtop pH-meter (Schwerzenbach, Switzerland), and was found to be 7.3.

\section{Crystalline solubility measurements}

The equilibrium solubilities of atazanavir and posaconazole were determined in all six media. An excess amount of crystalline solid was first equilibrated in each medium $(1 \mathrm{~mL})$ for 48 hours at $37^{\circ} \mathrm{C}$. Afterwards, the solubilized drug was separated by ultracentrifugation at 50,000 rpm $(135,700 \mathrm{~g})$ for 30 minutes, while 
maintained at $37^{\circ} \mathrm{C}$, using an Optima MAX-XP ultracentrifuge equipped with a Swinging-Bucket Rotor TLA-110 (Beckman Coulter, Inc., Brea, CA). For atazanavir, the supernatant concentration was determined by high performance liquid chromatography (HPLC) with a Hewlett Packard 1100 series system, using an Agilent Eclipse Plus C18 column ( $4.6 \mathrm{~mm} \times 250 \mathrm{~mm}, 5 \mu \mathrm{m})$ (Agilent Technologies, Santa Clara, CA). The mobile phase consisted of $60 \%$ water acidified to $\mathrm{pH} 2.5$ with o-phosphoric acid, and $40 \%$ acetonitrile. An injection volume of $20 \mu \mathrm{L}$ was used. The flow rate was set to $0.8 \mathrm{~mL} / \mathrm{min}$ where each analytical run was adjusted to $15 \mathrm{~min}$. Atazanavir was detected by ultraviolet (UV) absorbance at a wavelength of $247 \mathrm{~nm}$. Standards $(0.1-10 \mu \mathrm{g} / \mathrm{mL})$ were prepared in methanol, where the standard curve exhibited good linearity $\left(\mathrm{R}^{2}=0.999\right)$ over this concentration range. For posaconazole, an Agilent 1260 Infinity II HPLC system coupled with an Agilent Eclipse Plus C18 column ( $4.6 \mathrm{~mm} \times 250 \mathrm{~mm}, 5 \mu \mathrm{m})$ was used to analyze the concentration in the supernatant. The mobile phase was $60 \%$ acetonitrile and $40 \%$ deionized water. The analytical run duration was $6 \mathrm{~min}$, where the injection volume was $20 \mu \mathrm{L}$ and the mobile phase flow was held constant at $2.0 \mathrm{~mL} / \mathrm{min}$. Posaconazole was detected by fluorescence emission where the excitation wavelength was at $240 \mathrm{~nm}$ and the emission wavelength was at $385 \mathrm{~nm}$. Using posaconazole standards prepared in methanol, two calibration curves $\left(0.01-0.1 \mu \mathrm{g} / \mathrm{mL}, \mathrm{R}^{2}=0.997\right.$ and $\left.0.1-20 \mu \mathrm{g} / \mathrm{mL}, \mathrm{R}^{2}=1\right)$ were used to calculate the crystalline solubility in different media.

\section{Amorphous solubility measurements}

To measure the amorphous solubility, aliquots from an atazanavir DMSO stock solution $(50 \mathrm{mg} / \mathrm{mL})$ and a posaconazole methanol stock solution $(10 \mathrm{mg} / \mathrm{mL})$ were added to $1 \mathrm{~mL}$ of each medium, while stirred at $300 \mathrm{rpm}$ and maintained at $37^{\circ} \mathrm{C}$, to generate supersaturated solutions of both compounds where the total concentration exceeds the 'expected' amorphous solubilities, based on previous findings. ${ }^{36,41}$ To prevent crystallization, pre-dissolved HPMC-AS was added at a concentration of $10 \mu \mathrm{g} / \mathrm{mL}$ for atazanavir samples, and $100 \mu \mathrm{g} / \mathrm{mL}$ for posaconazole samples. Samples were then ultra-centrifuged using the same conditions used for the crystalline solubility measurements to pellet the drug-rich phase. The supernatant was separated from the precipitated drug-rich phase, diluted in methanol, and analyzed using the HPLC methodologies described above.

Confirmation of amorphous solubility and formation of LLPS nanodroplets by fluorescence spectroscopy and scanning electron microscopy (SEM)

To confirm the formation of colloidal aggregates which occurs when the amorphous solubility is exceeded, fluorescence spectroscopy was used to monitor the corresponding changes in the emission spectra of atazanavir and posaconazole. Both compounds are auto-fluorescent, and the emission spectrum varies depending on the hydrophobicity of the local environment, for example, whether the drug is molecularly 
dissolved or present in colloidal aggregates, as demonstrated previously. ${ }^{42-44}$ Fluorescence spectra were collected for both drugs at different concentrations, below and above the amorphous solubility, in the presence of HPMC-AS to prevent crystallization. Solutions were prepared via the solvent shift method as described before and maintained at $37^{\circ} \mathrm{C}$ while stirring at $300 \mathrm{rpm}$. Samples were analyzed using a Shimadzu RF- 5301pc Spectrofluorometer (Kyoto, Japan). The excitation wavelength used was $250 \mathrm{~nm}$ for atazanavir and $240 \mathrm{~nm}$ for posaconazole. The emission spectrum was collected at $0.2 \mathrm{~nm}$ intervals from 220 to $450 \mathrm{~nm}$. The excitation and emission slits width were kept at 5 or $10 \mathrm{~mm}$ depending on the medium and the intensity of the resulting emission spectra. Additionally, to further characterize the colloidal species formed in human fluids at concentrations above the amorphous solubility, SEM was utilized. This technique has been previously used to demonstrate the formation of the amorphous nanodroplets of cyanophenyl furanone dye in water ${ }^{13}$ and ritonavir in $\mathrm{pH} 6.8$ phosphate buffer. ${ }^{45}$ Briefly, highly supersaturated solutions of atazanavir and posaconazole above the measured amorphous solubility were prepared in FaHIF. 100 $\mu \mathrm{g} / \mathrm{mL}$ of HPMC-AS were added to the posaconazole sample to avoid crystallization. Samples were then centrifuged at 14,800 rpm for $10 \mathrm{~min}$, using a Sorvall Legend Micro 17 Centrifuge (Thermo Fisher Scientific, Waltham, MA). The supernatants were then discarded, and a small amount of the amorphous pellet was fixed on an SEM stub and sputter-coated with platinum for $60 \mathrm{~s}$. Imaging was performed with a FEI Teneo VS ${ }^{\mathrm{TM}}$ scanning electron microscope (FEI Company, Hillsboro, Oregon) in OptiPlan mode with $\mathrm{T} 1$ and $\mathrm{T} 2$ detectors to capture backscatter and secondary electron images. Operating conditions were $5 \mathrm{kV}$ accelerating voltage, $0.20 \mathrm{nA}$ current, and $\sim 2 \mathrm{~mm}$ working distance.

\section{Diffusion cell experiments}

A side-by-side diffusion cell (PermeGear Inc., Hellertown, PA) of $1.5 \mathrm{~mL}$ volume (each compartment) and $9 \mathrm{~mm}$ orifice diameter was used to determine the flux, $J$, at both the amorphous solubility $\left(J_{\text {amph }}\right)$ and the crystalline solubility $\left(J_{e q}\right)$ for each drug and medium. The donor and receiver compartments were separated by a regenerated cellulose membrane with a MWCO of 6-8KDa. Each compartment had $1 \mathrm{~mL}$ of media, was stirred using a magnetic stirrer $(2 \times 7 \mathrm{~mm})$ at $\sim 600 \mathrm{rpm}$ to minimize the thickness of the unstirred water layer, ${ }^{17}$ and was maintained at $37^{\circ} \mathrm{C}$. Using an atazanavir DMSO stock solution $(50 \mathrm{mg} / \mathrm{mL})$ and a posaconazole methanol stock solution $(10 \mathrm{mg} / \mathrm{mL})$, a concentration of each drug corresponding to the amorphous solubility for each medium, was added to the donor compartment to determine $J_{a m p h}$. To prevent crystallization, pre-dissolved HPMC-AS was added to both the donor and receiver compartments at concentrations of $10 \mu \mathrm{g} / \mathrm{mL}$ and $100 \mu \mathrm{g} / \mathrm{mL}$ for atazanavir and posaconazole experiments, respectively. Control experiments were performed with atazanavir in the absence of HPMC-AS in buffer-V1, demonstrating that the polymer had no effect on the mass flow rate. For determining $J_{e q}$, a suspension of crystalline drug, equilibrated for $48 \mathrm{~h}$ in each medium was added to the donor compartment. A $15 \mu \mathrm{L}$ aliquot 
was withdrawn from the receiver compartment at different timepoints, diluted in methanol, and analyzed by HPLC using the methods described above.

\section{Results}

\section{Crystalline solubility measurements}

The measured crystalline solubility values of atazanavir and posaconazole at $37^{\circ} \mathrm{C}$ in the six media are summarized in Table 2. Atazanavir crystalline solubilities were slightly higher in FaSSIF-V1, FaSSIF-V2, Composite-FaSSIF and FaHIF relative to in the two blank buffers. For posaconazole, the differences in the crystalline solubilities between both buffers and the other biorelevant media were more pronounced (7-14 fold) relative to atazanavir. This suggests that posaconazole was more effectively solubilized by the presence of mixed micelles in the media. Additionally, for posaconazole, the amount of lecithin influences the solubility, which was highest in FaSSIF-V1. Posaconazole solubilities in FaSSIF-V1, FaSSIF-V2 and FaHIF were in good agreement with values reported previously. ${ }^{41,46,47}$

Table 2. Crystalline solubility values of atazanavir and posaconazole in different media. $(n=3, \pm$ standard deviation).

Medium

Buffer-V1

Buffer-V2

FaSSIF-V1

FaSSIF-V2

Composite-FaSSIF

FaHIF

\section{Equilibrium solubility $(\mu \mathrm{g} / \mathrm{mL})$} Atazanavir

$1.4 \pm 0.2$

$1.5 \pm 0.3$

$2.7 \pm 0.1$

$2.6 \pm 0.4$

$3.1 \pm 0.6$

$7.1 \pm 0.6$

\section{Posaconazole}

$0.23 \pm 0.03$

$0.22 \pm 0.02$

$3.2 \pm 0.3$

$1.6 \pm 0.1$

$1.7 \pm 0.2$

$2.6 \pm 0.5$

\section{Amorphous solubility measurements}

Table 3 summarizes the measured amorphous solubilities of atazanavir and posaconazole in the six media at $37^{\circ} \mathrm{C}$ as determined by the ultracentrifugation method. As expected, the amorphous solubility was not notably different between buffer-V1 and buffer-V2 for either drug. In the presence of solubilizing species, atazanavir amorphous solubility increased by a factor of $\approx 1.25$. FaSSIF-V1, FaSSIF-V2, composite-FaSSIF and FaHIF yielded similar amorphous solubility values for atazanavir, suggesting the different configurations of bile salts/lecithin in these media had minimal effect on the solubilization of atazanavir in a supersaturated solution. This was not the case for posaconazole. Following the same trend as for the crystalline solubility, posaconazole amorphous solubility was highest in the medium with the greatest lecithin content (FaSSIF-V1). In FaSSIF-V2, the amorphous solubility value obtained was equivalent to 
our previous finding. ${ }^{41}$ Finally, the amorphous solubility appeared to be lower in composite-FaSSIF and FaHIF, compared to FaSSIF-V1 and FaSSIF-V2. Such differences between FaSSIF-V1/V2 and compositeFaSSIF have been observed previously for ritonavir, tacrolimus and cilnidipine. ${ }^{36}$

Table 3. Amorphous solubility values in atazanavir and posaconazole in different media. $(n=3, \pm$ standard deviation).

\begin{tabular}{ccc} 
Medium & \multicolumn{2}{c}{$\begin{array}{c}\text { Amorphous solubility }(\boldsymbol{\mu g} / \mathbf{m L}) \\
\text { Ptazanavir }\end{array}$} \\
Buffer-V1 & $79 \pm 4$ & $8.1 \pm 0.2$ \\
Buffer-V2 & $82 \pm 1$ & $8.2 \pm 0.3$ \\
FaSSIF-V1 & $102 \pm 3$ & $43.3 \pm 2.3$ \\
FaSSIF-V2 & $101 \pm 10$ & $20.8 \pm 1.6$ \\
Composite-FaSSIF & $100 \pm 3$ & $15.5 \pm 0.7$ \\
FaHIF & $99 \pm 2$ & $13.2 \pm 0.4$
\end{tabular}

Figure 2 shows the fluorescence emission spectra as a function of atazanavir/posaconazole concentration in the different media. When the amorphous drug-rich phase was formed at concentrations above the amorphous solubility, an increase in the peak intensity was observed. The concentration where the increase in intensity occurred showed good correspondence to the amorphous solubility values measured by the ultra-centrifugation method (Table 3). Additionally, some samples exhibited a shift in the peak maximum to shorter wavelengths (blue shift). This is attributed to the decrease in local polarity experienced by the drug molecules when colloidal species form. As a result, the quantum yield increases and the emission maximum shifts to shorter wavelengths (hypsochromic shift).

The size and morphology of the atazanavir and posaconazole colloidal species formed in FaHIF is illustrated by the SEM images shown in Figure 3. Amorphous aggregates for both drugs were mostly spherical and clearly different from crystalline material, such as atazanavir crystals which are needleshaped. ${ }^{48,49}$ 
Atazanavir
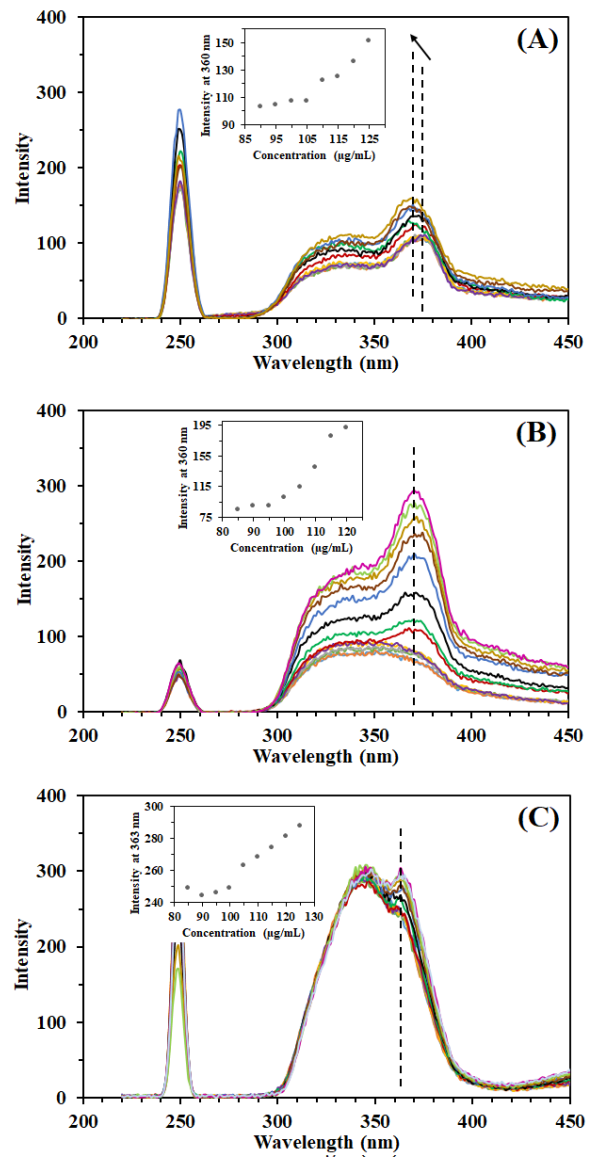

B

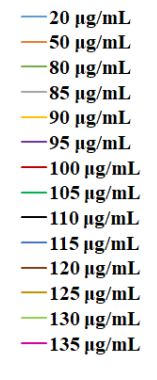

$-50 \mu \mathrm{g} / \mathrm{mL}$

$-50 \mu \mathrm{g} / \mathrm{mL}$
$-75 \mu \mathrm{g} / \mathrm{mL}$

$-75 \mu \mathrm{g} / \mathrm{mL}$
$-80 \mu \mathrm{g} / \mathrm{mL}$

$-85 \mu \mathrm{g} / \mathrm{mL}$

$-85 \mu \mathrm{g} / \mathrm{mL}$
$-90 \mu \mathrm{g} / \mathrm{mL}$

$-95 \mu \mathrm{g} / \mathrm{mL}$

$-100 \mu \mathrm{g} / \mathrm{mL}$

$-105 \mu \mathrm{g} / \mathrm{mL}$

$-110 \mu \mathrm{g} / \mathrm{mL}$

$-115 \mu \mathrm{g} / \mathrm{mL}$

$-120 \mu \mathrm{g} / \mathrm{mL}$

$-125 \mu \mathrm{g} / \mathrm{mL}$

$-130 \mu \mathrm{g} / \mathrm{mL}$

$-135 \mu \mathrm{g} / \mathrm{mL}$
$-140 \mu \mathrm{g} / \mathrm{mL}$
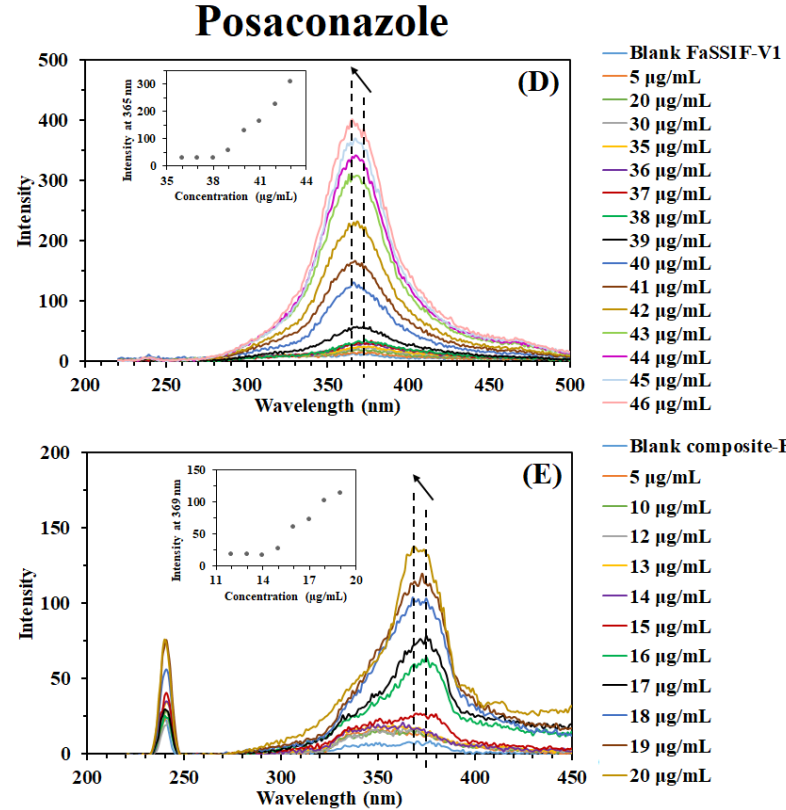

(E)

- Blank composite-FaSSIF

$-5 \mu \mathrm{g} / \mathrm{mL}$

$-10 \mu \mathrm{g} / \mathrm{mL}$

$-12 \mu \mathrm{g} / \mathrm{mL}$

$-13 \mu \mathrm{g} / \mathrm{mL}$

$-14 \mu \mathrm{g} / \mathrm{mL}$

$-15 \mu \mathrm{g} / \mathrm{mL}$

$-16 \mu \mathrm{g} / \mathrm{mL}$

$-17 \mu \mathrm{g} / \mathrm{mL}$

$-18 \mu \mathrm{g} / \mathrm{mL}$

$-19 \mu \mathrm{g} / \mathrm{mL}$

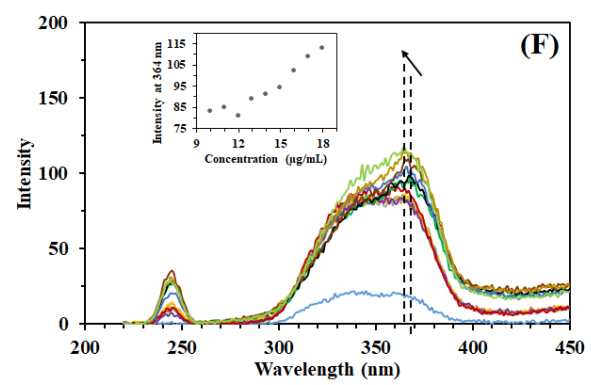

- Blank FaHIF

$-5 \mu \mathrm{g} / \mathrm{mL}$

$-8 \mu \mathrm{g} / \mathrm{mL}$

$-10 \mu \mathrm{g} / \mathrm{mL}$

$-11 \mu \mathrm{g} / \mathrm{mL}$

$-12 \mu \mathrm{g} / \mathrm{mL}$

$-13 \mu \mathrm{g} / \mathrm{mL}$

$-14 \mu \mathrm{g} / \mathrm{mL}$

$-15 \mu \mathrm{g} / \mathrm{mL}$

$-16 \mu \mathrm{g} / \mathrm{mL}$

$-17 \mu \mathrm{g} / \mathrm{mL}$

$-18 \mu \mathrm{g} / \mathrm{mL}$

Figure 2. Fluorescence emission spectra of atazanavir (left side) and posaconazole (right side) at different concentrations in (A), (D) FaSSIF-V1, (B), (E) composite-FaSSIF, and (C) and (F) FaHIF. The arrows and vertical dashed lines illustrate a blue shift in the peak maximum for some samples. The inset illustrates the increase in intensity as a function of concentration where the change of slope corresponds to the formation of the second phase of amorphous nanodroplets. 

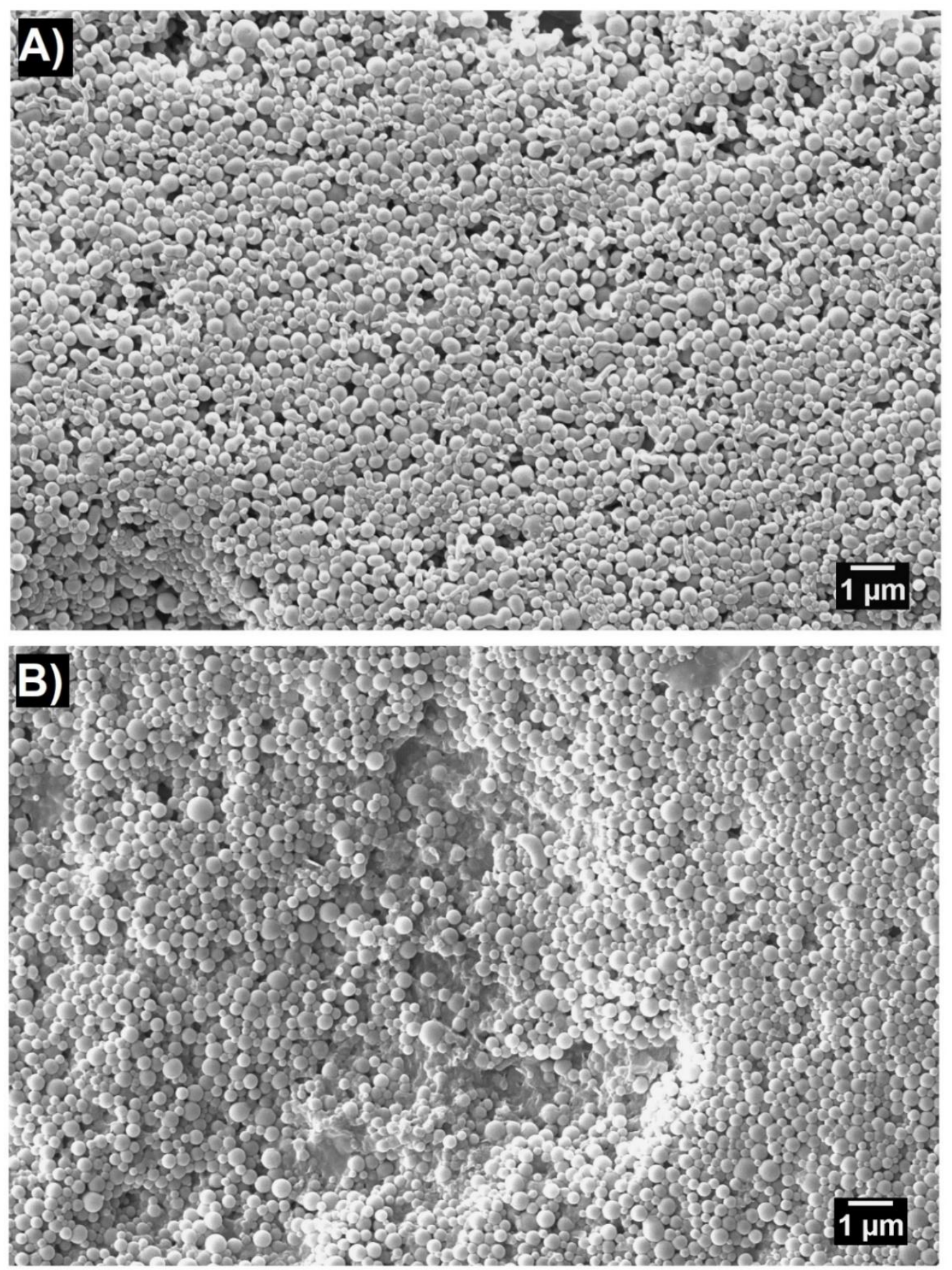

Figure 3. SEM images of A) atazanavir and B) posaconazole amorphous nanodroplets precipitated from FaHIF. Posaconazole nanodroplets were stabilized by HPMC-AS to prevent crystallization.

\section{Supersaturation determination by diffusive flux measurements}

The supersaturation ratio $(S R)$ was determined using the ratio of the thermodynamic activity of the solute in the solution, $a$, to the activity of the solute at its crystalline state, $a_{e q}$, as shown in Equation 2. The activity 
can be described in terms of $\gamma$, the activity coefficient of the drug in the solution and $C$, the drug concentration, where the subscript, $e q$, refers to the property at equilibrium.

$S R=\frac{a}{a_{e q}}=\frac{\gamma C}{\gamma_{e q} C_{e q}}$

Solute activity at the crystalline and amorphous solubilities can be assessed using diffusion cell experiments to determine the diffusive flux, $J$, across a semipermeable membrane, as shown in Equation 3:

$J=\frac{D a}{h \gamma_{m}}(3)$

where $D$ is the diffusion coefficient of the solute, $h$ is the thickness of the membrane and $\gamma_{m}$ is the activity coefficient of the solute in the membrane, which are all assumed to be constants for a particular drug and medium. Consequently, the maximum attainable supersaturation, $S R_{\max }$, in a given medium can be derived from the ratio of the diffusive flux of the solute at the amorphous solubility, $J_{a m p h}$, to the diffusive flux at the crystalline solubility, $J_{e q}$, as demonstrated in Equation 4:

$S R_{\max }=\frac{J_{a m p h}}{J_{e q}}$

In deriving Equation 4, we assume that the thermodynamic activity of the solute is the same as the thermodynamic activity of the second phase present in the system; this is either crystalline or amorphous drug. To achieve a well-defined activity, we therefore measure the flux of systems containing a suspension of either crystalline or amorphous drug. The impact of different media on mass transport and maximum supersaturation level was studied using diffusion cell experiments performed at both the amorphous and crystalline solubilities at $37^{\circ} \mathrm{C}$. The mass transport time-profiles and $J$ (derived from the slope of the concentration versus time profiles) values for atazanavir and posaconazole in buffer-V1, composite-FaSSIF and FaHIF are shown in Figures 4 and 5, respectively. For both compounds, $J_{a m p h}$ and $J_{e q}$ were relatively close between the different media. All normalized flux values for atazanavir and posaconazole are shown in Table S1 (supporting information). Additionally, the apparent permeability values were calculated accordingly and are shown in Tables $\mathbf{S 2}$ and S3. 


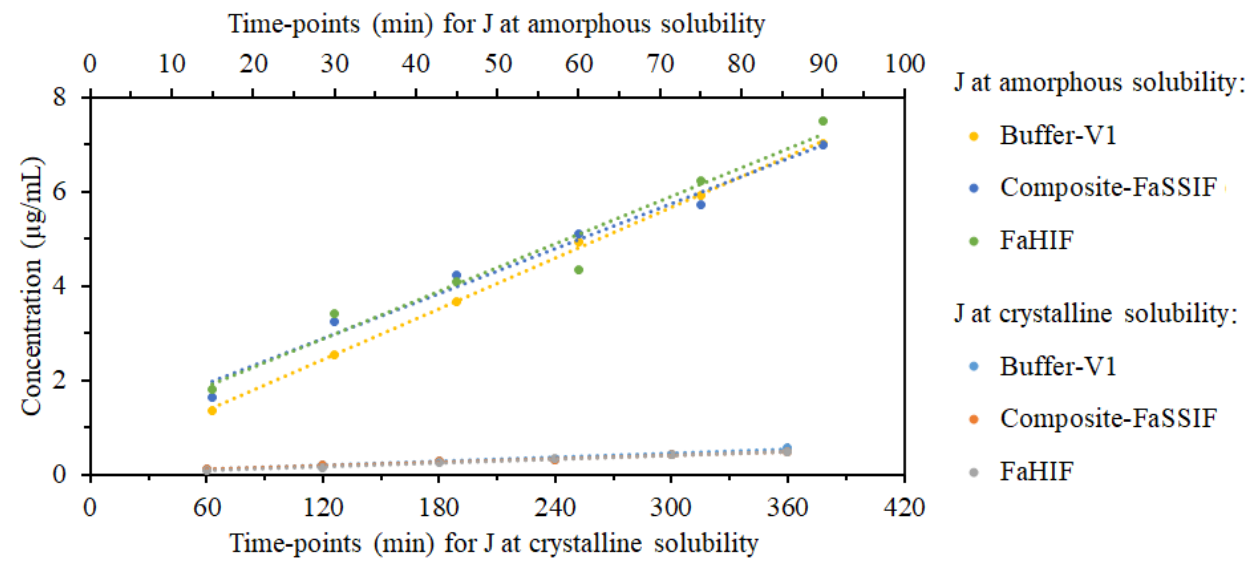

Figure 4. Atazanavir mass transport versus time at the amorphous solubility (upper $\mathrm{x}$-axis) and at the crystalline solubility (lower $\mathrm{x}$-axis), in different media. Error bars were omitted for clarity. $(\mathrm{n}=3)$.

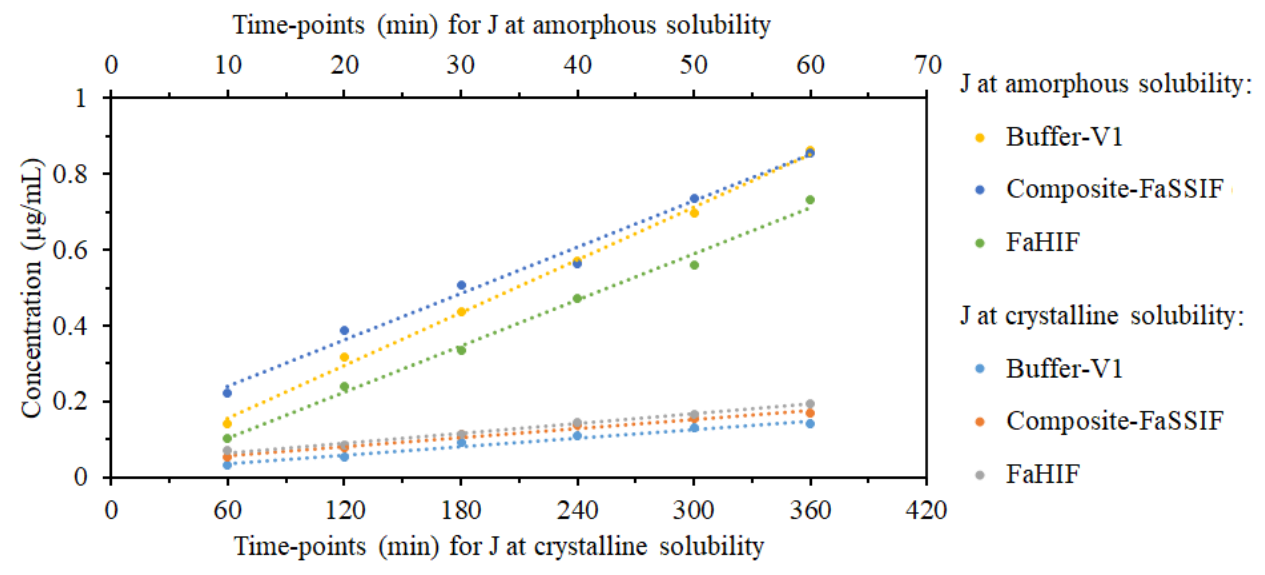

Figure 5. Posaconazole mass transport versus time at the amorphous solubility (upper $\mathrm{x}$-axis) and at the crystalline solubility (lower $\mathrm{x}$-axis), in different media. Error bars were omitted for clarity. (n=3).

Figures 6 and 7 show the $S R_{\max }$ determined in different media for atazanavir and posaconazole, respectively, based on Equations 1 (concentration-based) and 4 (activity-based). For both compounds, large differences between the concentration- and activity-based $S R_{\max }$ are apparent for some media. The concentration-based $S R_{\max }$ is the ratio of the amorphous and crystalline solubilities for the various media, (i.e. the ratio of the values presented in Tables 2 and 3), while the activity-based values are calculated from the ratio of the flux measured for a crystalline and amorphous suspension in the different media (i.e. the ratio of the values presented in Figures 4 and 5). While $S R_{\max }$ was relatively consistent between the two approaches for the aqueous buffers, the concentration-based $S R_{\max }$ values decreased in all other biorelevant media and was lowest in aspirated FaHIF. Thus, if the concentration-based $S R_{\max }$ is considered, the maximum achievable supersaturation appears to decrease in FaHIF. In contrast, the activity-based $S R_{\max }$ ratios were approximately the same across the various media types. For atazanavir, the activity-based $S R_{\max }$ averaged $\sim 54$ between the different media, where no values were significantly different from the value 
measured for FaSSIF-V1 (based on two-tailed t-test, $\mathrm{p}>0.05$ ). Therefore, all media achieved an equivalent maximum supersaturation for atazanavir. Indulkar et al. previously reported an average activity-based supersaturation of $65 .^{36}$ The activity-based $S R_{\max }$ was found to be $\sim 36$ for posaconazole. Although the values were generally similar for different media, a slight decrease was noticed for the activity-based $S R_{\max }$ for posaconazole in both composite-FaSSIF and FaHIF (based on two tailed t-test, $\mathrm{p}<0.05$ ).

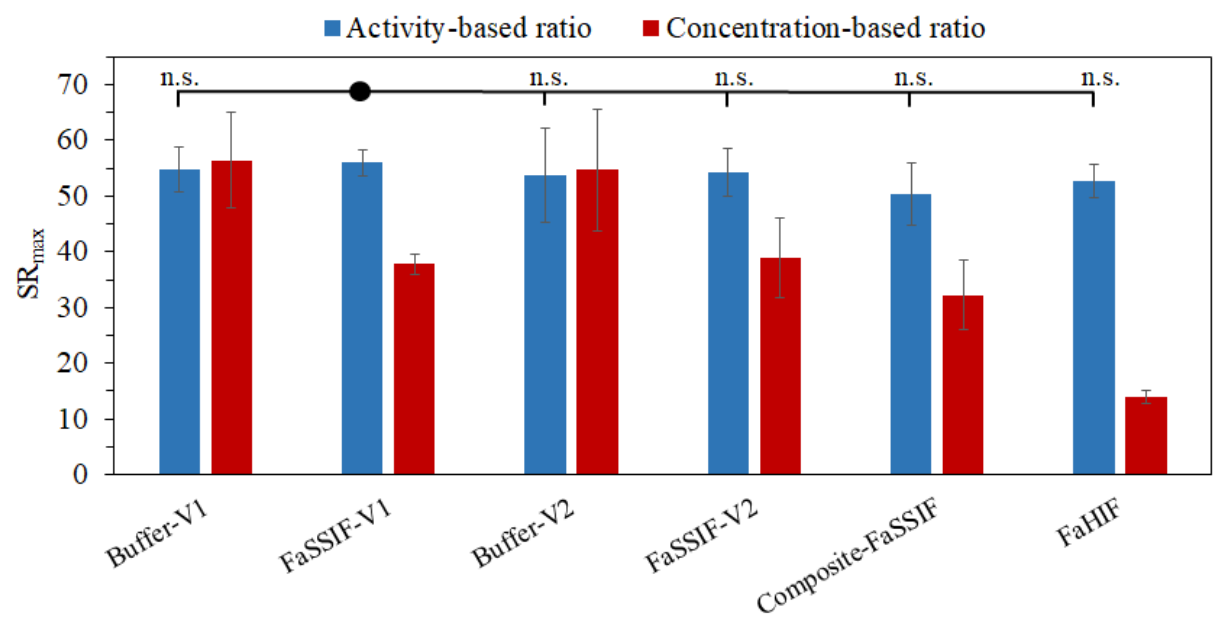

Figure 6. Maximum supersaturation ratio for atazanavir in different media obtained using activity-based ratio (blue bars) versus concentration-based ratio (red bars). The n.s. indicates when the difference between FaSSIF-V1 and other datasets was nonsignificant ( $\mathrm{t}$-test, $\mathrm{p}$ value $>0.05$ ). Error bars represent standard deviations where $\mathrm{n}=3$.

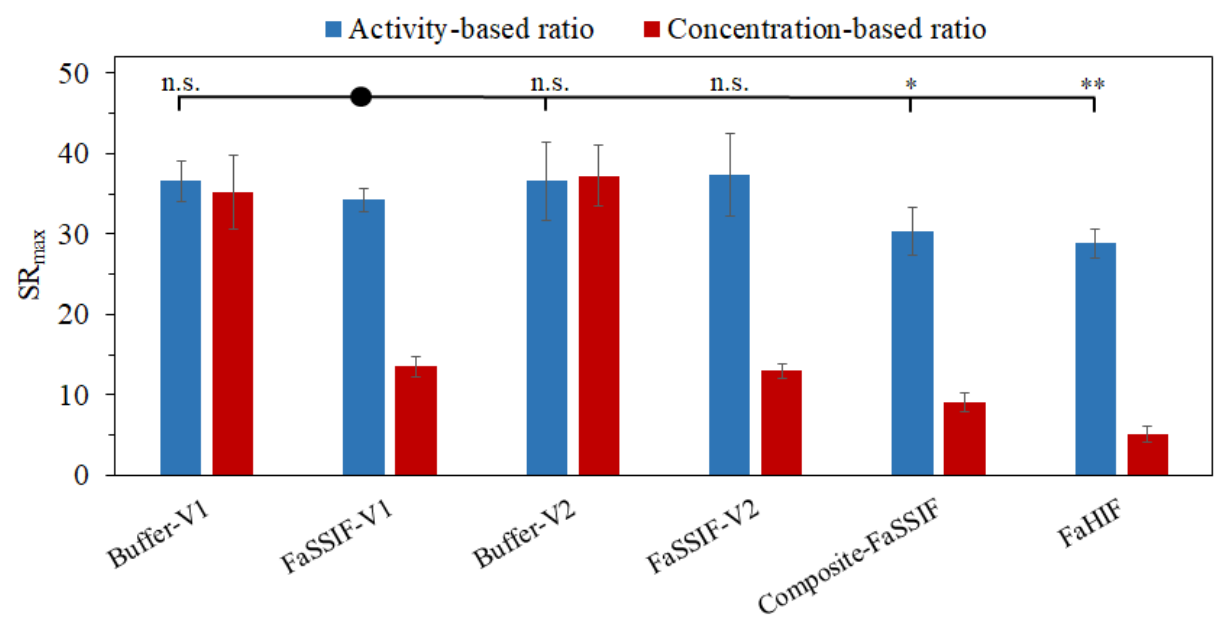

Figure 7. Maximum supersaturation ration for posaconazole in different media obtained using activity-based ratio (blue bars) versus concentration-based ratio (red bars). The n.s. indicates when the difference between FaSSIF-V1 and other datasets was non-significant ( $\mathrm{t}$-test, $\mathrm{p}$ value $>0.05$ ), whereas the $*$ and $* *$ symbols indicate that the difference was statistically significant (t-test, $\mathrm{p}$ value $=0.002$ and $5.3 \times 10^{-7}$, respectively). Error bars represent standard deviations where $n=3$.

\section{Discussion}


Supersaturation is considered an important factor impacting the extent of oral absorption of drugs with solubility-limited absorption. ${ }^{4,50}$ Supersaturation can be generated via a variety of routes, including $\mathrm{pH}$ increase following gastrointestinal transit, which is of relevance for weakly-basic drugs, and by release of drugs formulated as amorphous solid dispersions. Understanding the extent and duration of supersaturation of a particular drug is critical to predict its absorption rate in vivo. This statement is based on the well-known relationship, derived from Fick's first law, shown in Equation 5:

$J=P C(5)$

where $J$ is the flux across a biological membrane, $P$ is the membrane permeability and $C$ is drug concentration. This relationship implies that an increase in drug concentration beyond the crystalline solubility will lead to an increase in the transport rate across a membrane, and, in the absence of confounding factors such as efflux and first pass metabolism, will increase the absorption rate. Several studies have shown a linear relationship between the membrane flux and supersaturation in simple media, ${ }^{6,10,51}$ which is truncated when the amorphous solubility is reached. Thus, as the concentration of the drug is increased (supersaturation is increased), the thermodynamic activity of the drug increases until it reaches its maximum at the amorphous solubility and phase separation to an amorphous phase occurs. ${ }^{10}$ This leads to the formation of a drug-rich colloidal phase in metastable equilibrium with a supersaturated solution. In the present study, the formation of such colloidal species in FaHIF, upon exceeding the amorphous solubility, is clearly demonstrated for the first time (Figure 3). Given that these colloidal species are thought to be advantageous for passive diffusion in vivo by maintaining the supersaturation at the maximum value through their reservoir effect, ${ }^{52}$ this is a significant finding.

In contrast to the relationships established in simple media, the results shown in Figures 6 and $\mathbf{7}$ clearly suggest that using concentration ratios to determine supersaturation for the lipophilic, un-ionized compounds studied, is inaccurate in complex media given the absence of a linear relationship between solute concentration and membrane flux. These observations are of profound importance since concentration profiles are so routinely used to make predictions about in vivo formulation performance. Therefore, it is important to understand where the limitations arise with respect to the use of concentrationbased measurements in complex media.

To explain the observed differences between total dissolved concentration- and activity-based measurements (Figures 6 and 7), it is important to first differentiate solubilization from supersaturation, and then to consider the interplay between the two for different media. Both phenomena result in an increase in the 'apparent' concentration of the drug in solution, yet have different thermodynamic consequences in terms of membrane mass transport rate. Supersaturation results in an increase in the 'free' drug 
concentration in solution relative to a saturated/equilibrium state. Consequently, solute thermodynamic activity (i.e. the effective concentration) is higher (Equation 2), thus the membrane transport is increased (Equation 3). Solubilization occurs through the presence of solubilizing agents that are either present naturally such as the biological surfactants (bile salts) or introduced as formulation excipients such as synthetically derived surfactants or complexing agents. ${ }^{53}$ In intestinal fluids (simulated and real), micelles and other colloidal species, formed by bile salts and lecithin, provide a microphase with which drug molecules can associate by binding with surfactant or by incorporation into the micellar core. These additional solution species lead to a dynamic equilibrium between at least two populations of drug molecules, those associated with the micelles, and free drug molecules in solution. Thus, increases in equilibrium solubility are observed (as seen in Table 2), whereby the solute activity remains constant since it is dictated by the crystalline form of the drug in equilibrium with the solution phase. Thus, solubilization of this system does not increase the 'free' drug concentration (i.e. solute thermodynamic activity) in solution, although the total concentration measured is increased. As described in the literature, membrane transport rates do not correlate with total dissolved concentration in the presence of solubilizing species. ${ }^{35,54-57}$ This is because for small molecules, only the 'free' unionized drug will undergo membrane partitioning and transport. ${ }^{58}$ We see the same outcome in the current study, where the flux values at the crystalline solubility do not show any correlations with increases in the equilibrium solubility due to solubilization (Figures 4 and $\mathbf{5}$ ).

The complexity of the situation increases considerably when both supersaturation and solubilization occur. To assess the supersaturation ratio in the presence of solubilizing components, Equation 1 is typically employed. ${ }^{4,40,59-61}$ Here, the crystalline solubility is measured in the medium of interest for the supersaturation determination to take into account the solubilization effect. For example, crystalline solubility has been determined in partially digested lipid media, ${ }^{59,62-64}$ or intestinal fluids collected at different time points following oral dosing..$^{40,65-68}$ The assumption implicit in this approach is that the fraction of the drug that is solubilized at the crystalline solubility is the same as the fraction of the drug that is solubilized in the supersaturated solution. Thus, the solubilization effect is cancelled by taking the ratio of the two values. This can be seen mathematically from Equation 2. If the ratio $\gamma / \gamma_{e q}$ (Equation 2) is 1, a situation that would arise when the extent of solubilization is the same over the concentration range of interest and there are no other non-idealities, then Equation 1 reasonably describes the true supersaturation. However, if the solubilization capacity varies as a function of concentration and supersaturation, then $\gamma / \gamma_{e q}$ $\neq 1$, and Equation 1 will not accurately describe the supersaturation of the system. ${ }^{43}$ This appears to be the situation in the biorelevant media tested herein and warrants consideration of the solubilization capacity of the various media at different supersaturations. 
Interestingly, our in vitro studies appear to be in good agreement with observations made following oral dosing of posaconazole and subsequent investigations of supersaturation evolution. Posaconazole is a highly permeable compound with solubility-limited absorption. ${ }^{69}$ Hens et al. conducted a study to investigate posaconazole supersaturation in vivo in the small intestine upon administration of an acidified/neutral posaconazole suspension, ${ }^{40}$ or a posaconazole solid dispersion. ${ }^{67}$ Here, samples were collected from the intestine and the supersaturation was determined by measuring the posaconazole solution concentration, and the crystalline solubility in the individual fluid samples, i.e. a concentration-based supersaturation measurement as per Equation 1. In the first study with suspensions, the mean maximum supersaturation ratio observed was $\approx 4$, whereas the results of the second study with amorphous solid dispersions showed a mean maximum supersaturation of $\approx 7$ upon fasted-state administration. Remarkably, these values are in close agreement with our FaHIF concentration-based supersaturation assessment at the amorphous solubility limit, which is $\approx 5$ (Figure 6). Notably, our study shows that the solution in FaHIF at the amorphous solubility displays a 30 -fold increase in mass transport rate relative to a crystalline suspension, indicating that the amorphous solubility advantage is not lost in FaHIF, despite the apparent reduction in the concentration-based supersaturation ratio. This once again illustrates the necessity of using activity-based assessments to avoid underestimation of supersaturation and suggests that the solutions generated in vivo in the previous studies were likely much more highly supersaturated than they appeared based on the concentration-based measurement approach employed.

A simple model for describing solubilization capacity of a given surfactant for a particular drug is to apply the concept of a micellar partition coefficient $\left(K_{m / w}\right)$, which is given by Equation 6: ${ }^{53}$

$K_{m / w}=\frac{C_{m}}{C_{w}}(6)$

Where $C_{m}$ is the surfactant-associated drug concentration, and $C_{w}$ is the free drug concentration. While this model is likely oversimplified for a complex system of bile acids and lecithin, it serves to illustrate how the extent of solubilization may vary as a function of concentration and supersaturation. In biorelevant media, solubilizing components interact with the solute of interest, whereby the interactions vary depending on the level of supersaturation. Specifically, mixed bile salt-lecithin micelles exist in the test fluids and these can solubilize drugs. ${ }^{28,29}$ We can estimate the micelle partition coefficient at the amorphous and crystalline solubilities using Equation 6. Here, the denominator is taken as the solubility in buffer, where only free drug is present. Assuming that the solubilizing components do not mix with either the crystalline or amorphous phase, then the free drug concentration is the same in the complex media as for buffer (since it is determined by the thermodynamic properties of the second phase present, i.e. either crystalline or watersaturated amorphous drug). The excess concentration of drug relative to buffer is therefore associated with 
the solubilizing species, enabling determination of $K_{m / w} . K_{m / w}$ values for both compounds in FaSSIF-V1, composite-FaSSIF and FaHIF, are shown in Table 4. Evaluating the $K_{m / w}$ values for the crystalline solubilities, we note that posaconazole has a higher value than atazanavir indicating that this compound is more effectively solubilized by the micelles. Of particular interest, we note that $K_{m / w}$ is much lower at the amorphous solubility than at the crystalline solubility for both compounds. This observation is in agreement with the result reported by Indulkar et al. for atazanavir and sodium dodecyl sulfate systems, ${ }^{43}$ where $K_{m / w}$ was observed to be highly concentration dependent. Of further note, is the observation that $K_{m / w}$ varies as a function of medium, reflecting a difference in their solubilization capacity. Of final note, $K_{m / w}$ is dependent on the drug, i.e. posaconzole had higher solubilization capacities than atazanavir, which explains why the discrepancies in supersaturation ratios were more prominent for posaconazole (Figure 7) compared to atazanavir (Figure 6).

Table 4. Estimation of $K_{m / w}$ values for atazanavir and posaconazole at the crystalline and amorphous solubilities in FaSSIF-V1, composite-FaSSIF and FaHIF media.

\begin{tabular}{|ccccc|} 
Medium & \multicolumn{2}{c}{$\boldsymbol{K}_{\boldsymbol{m} / \boldsymbol{w}}$ at crystalline solubility } & \multicolumn{2}{c}{$\boldsymbol{K}_{\boldsymbol{m} / \boldsymbol{w}}$ at amorphous solubility } \\
& Atazanavir & Posaconazole & Atazanavir & Posaconazole \\
FaSSIF-V1 & 1.9 & 13.9 & 1.3 & 5.3 \\
Composite-FaSSIF & 2.2 & 7.4 & 1.3 & 1.9 \\
FaHIF & 5.1 & 11.3 & 1.3 & 1.6 \\
\hline
\end{tabular}

In examining the data shown in Table $\mathbf{2}$ and $\mathbf{3}$, it is clear that the crystalline solubility showed a proportionally greater increase in the presence of bile salts and lecithin in comparison to the amorphous solubility, for both compounds. For instance, the crystalline solubility for posaconazole in FaSSIF-V1 is $\approx$ 14 times that in buffer-V1, while the amorphous solubility in FaSSIF-V1 is $\approx 5$ times that in buffer-V1. Moreover, posaconazole exhibited a notably lower amorphous solubility in composite-FaSSIF and FaHIF compared to commercial FaSSIF media. This can be attributed in large part to the change in $K_{m / w}$ between these media (Table 4) given that these solutions show similar membrane flux values. In other words, the free drug concentration, and thus the supersaturation, remains similar between the different media, as evidenced by the similar membrane transport rates (Figure 7). Thus, the concentration-based supersaturation ratio drastically underpredicts the posaconazole membrane flux for micelle-containing media.

Figure 8 displays a schematic illustration of posaconazole micellar solubilization in FaSSIF-V1, composite-FaSSIF and FaHIF, in solutions saturated with respect to the crystalline or amorphous forms. This schematic illustrates the concept that the free drug concentration remains constant for these conditions. On the other hand, the amount of drug associated with the micelle varies depending on the composition of 
the solubilizing species, which can be attributed to micelles with different properties. We can anticipate that for a given medium, the constituent micelles have a finite capacity to solubilize the drug molecules. Therefore, the decrease in $K_{m / w}$ at the amorphous solubility relative to the crystalline solubility, reflects the reduced ability of the micelles to accommodate the increased number of molecules in solution. This clearly varies between the different media; there is a notable increase in posaconazole amorphous solubility when more lecithin was present in the medium i.e. FaSSIF-V1. This indicates that this system can solubilize a relatively higher proportion of posaconazole molecules in the micelles, leading to a higher $K_{m / w}$ value relative to the other media. Lecithin is a mixture of poorly-soluble phospholipids that can interact with bile salts forming mixed micelles. ${ }^{29,70}$ These mixed micelles with greater lecithin content clearly improve posaconazole solubilization in supersaturated solutions. The same was not true for atazanavir. Atazanavir amorphous solubility values were largely unaffected in different media. While atazanavir and posaconazole have similar Log P values, their chemical structures are quite different. Hammad and Muller explored the differences in solubility of various drugs in bile salt phosphatidylcholine-based mixed micelles. ${ }^{71}$ Their results showed that the extent of solubilization was governed by several factors including the size of the mixed micelles, as well as the drug molecular size, chain branching, polarizability and structural conformations. ${ }^{71}$ Therefore, the difference in the extent of solubilization for posaconazole and atazanavir likely arises from structural differences between these two compounds. For the different media, we note that $K_{m / w}$ undergoes the largest decrease between the crystalline and amorphous solubilities for FaHIF. This means that using the concentration-based supersaturation ratio can be expected to lead to the largest discrepancies in the prediction of the membrane mass transport rates when attempting to evaluate supersaturation in this medium. 


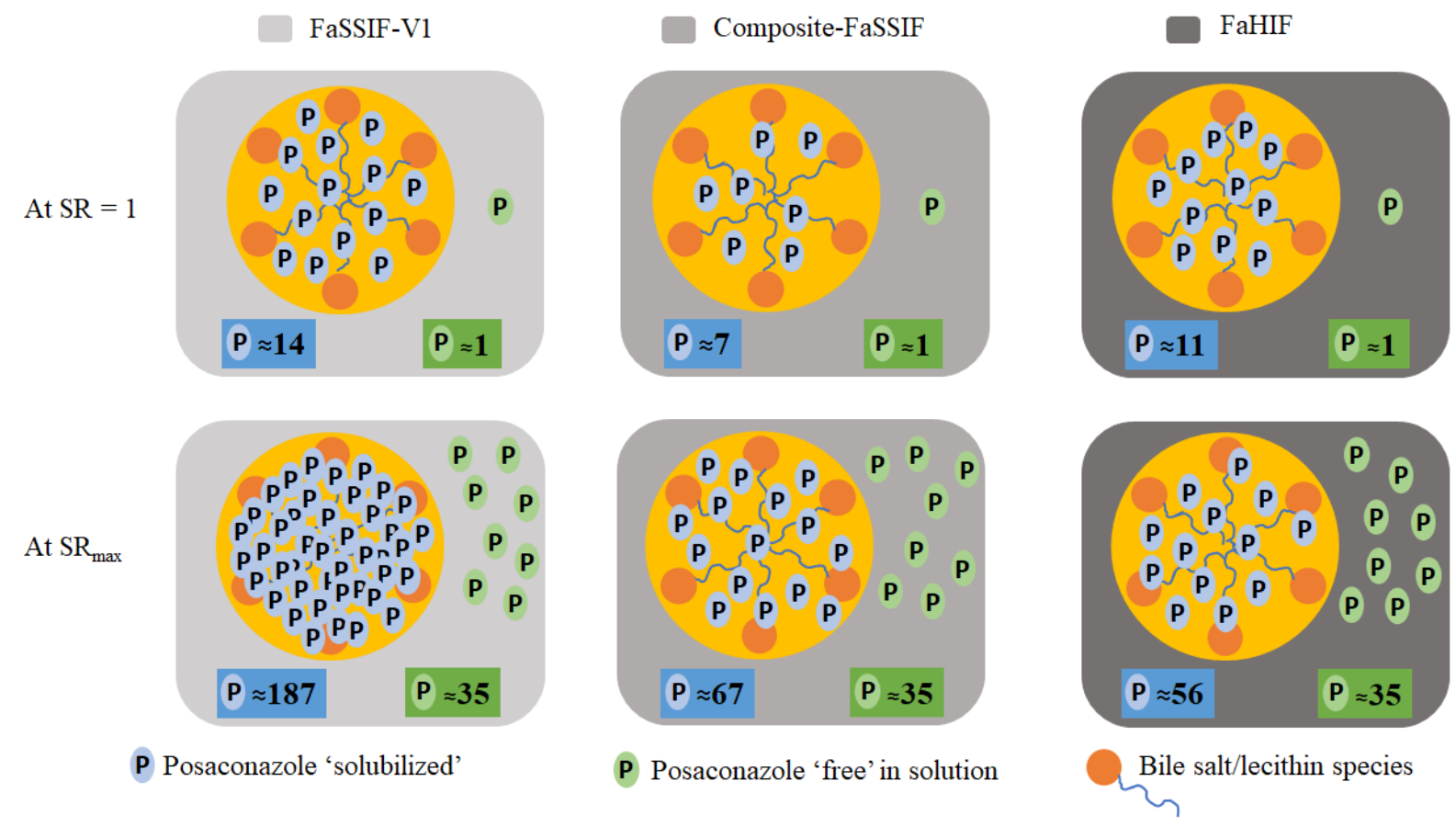

Figure 8. Schematic illustration of posaconazole micellar partitioning at saturation (crystalline solubility or SR=1) and maximum supersaturation (amorphous solubility or $\mathrm{SR}_{\max }$ ) levels. The numbers provide the ratio of the drug molecules in the micelle (blue box) to the free drug in solution (green box). For $\mathrm{SR}_{\max }$ (lower panel), the count of 'P' molecules is scaled down for clarity.

Based on our observations, it is clearly important to carefully reconsider the appropriateness of concentration-based ratios for evaluation of the supersaturation level. It is undoubtedly more accurate to use the thermodynamic activity-based ratio in complex media with bile salts, albeit more complex from an experimental perspective. ${ }^{72}$ In the absence of micelles, e.g. in a simple aqueous buffer, there is no solubilization effect and therefore the drug concentration in solution reasonably represents the free drug concentration, leading to a good agreement between the activity-based ratio and the concentration-based ratio in the buffer systems, as seen for both compounds (Figures 4 and 5). In this instance, the activity coefficient ratio $\left(\gamma / \gamma_{e q}\right)$ of the drug in solution is close to 1 (Equation 2), due to the very dilute nature of the system whereby solution non-idealities are minimized. The differences in the degree of discrepancy for concentration vs. activity-based measurements for the two compounds as a function of media type correlates with the difference in the solubilization capacity for each drug. The activity-based $S R_{\max }$ values on the other hand were fairly consistent for atazanavir and posaconazole in all media. This was expected since the supersaturation level is largely independent of the medium used as the thermodynamic activity-based boundaries remain unchanged. It should be noted that for posaconazole, there was statistically significant decrease in activity-based $S R_{\max }$ in composite-FaSSIF and FaHIF. The slight reduction in flux at the amorphous solubility for FaHIF and composite-FaSSIF (Figure 7) for posaconazole could be due to mixing of specific bile salts with the amorphous posaconazole phase, which can reduce the free drug concentration 
in equilibrium with the drug-rich phase. ${ }^{32}$ This was observed previously for telaprevir in the presence of STCDC and SGCDC dihydroxy bile salts, where the thermodynamic $S R_{\max }$ decreased to about three-fourths of the value in the absence of these bile salts. ${ }^{32}$ Both composite-FaSSIF and FaHIF contain STCDC and SGCDC, and the slight decrease in $J_{\text {amph }}$ values in these media could arise via a similar mechanism as reported for telaprevir. Our observations have important implications regarding the interplay between solubilization and supersaturation as a function of concentration. The most important of these is that Equation 1 cannot be used to estimate supersaturation in complex biorelevant media or aspirated human fluids, in particular for compounds showing solubilization. Using Equation 1, supersaturation would be underestimated, as seen in Figures 6 and 7, and the amorphous solubility advantage would seem to decrease in vivo.

Composite-FaSSIF, containing six bile salts, more closely mimics the human bile composition relative to commercial FaSSIF recipes, which contains only STC. Simulating the FaHIF composition may be especially important in the context of intraluminal dissolution, supersaturation and absorption kinetics of supersaturating formulations. Nevertheless, a high degree of inter- and intra-subject variability is reported in the literature for FaHIF in terms of both composition and the absolute concentrations of the constituent components. ${ }^{20}$ Additionally, other components are usually present in FaHIF at low concentration which are not present in composite-FaSSIF. These include cholesterol ${ }^{20,73,74}$ as well as lipid degradation products, ${ }^{20,75}$ such as fatty acids and monoacylglycerides. Although composite-FaSSIF comes closer to simulating FaHIF in terms of solubilization and supersaturation behavior relative to the commercially available media, more work needs to be done in order to optimize simulated fluids for in vitro testing of supersaturating formulations.

\section{Conclusion}

To improve the biopredictability of supersaturating formulations, it is important to understand the impact of different media composition on solubility and solution thermodynamics. Herein, we demonstrated that crystalline and amorphous solubility values were greatly influenced by the solubilization capacity of each medium for two un-ionized, lipophilic weakly basic compounds. This resulted in different micellar partitioning between the solubilized and solution phases among the different media and reduced apparent supersaturation ratios based on concentration measurements. However, since the effective drug concentration is approximately the same in all media, as shown by the flux measurements, the activitybased supersaturation was relatively independent of the media. These results provide the framework for further studies into the impact of different media on the phase behavior of supersaturated solutions.

\section{Acknowledgements}


Danny Riethorst is acknowledged for assisting in collection and handling of the human intestinal fluids. The authors would like to thank Yoon Yeo and Maie S. Taha for facilitating and assisting in the ultracentrifugation experiments. The authors acknowledge the National Science Foundation for their financial support for this research through research grants CHE-1412888 and DMR-1309218.

\section{Supporting Information}

Table S1 shows the normalized diffusive flux values, derived from the slope of mass transport rates of atazanavir and posaconazole. Table $\mathbf{S 2}$ and $\mathbf{S 3}$ show the calculated apparent permeability values for both compounds. 


\section{References}

(1) Loftsson, T.; Brewster, M. E. Pharmaceutical Applications of Cyclodextrins: Basic Science and Product Development. J. Pharm. Pharmacol. 2010, 62 (11), 1607-1621.

(2) Williams, H. D.; Trevaskis, N. L.; Charman, S. A.; Shanker, R. M.; Charman, W. N.; Pouton, C. W.; Porter, C. J. H. Strategies to Address Low Drug Solubility in Discovery and Development. Pharmacol. Rev. 2013, 65 (1), 315-499.

(3) Müllertz, A.; Ogbonna, A.; Ren, S. New Perspectives on Lipid and Surfactant Based Drug. J. Pharm. Pharmacol. 2010, 62 (11), 1622-1636.

(4) Brouwers, J.; Brewster, M. E.; Augustijns, P. Supersaturating Drug Delivery Systems: The Answer to Solubility-Limited Oral Bioavailability? J. Pharm. Sci. 2009, 98 (8), 2549-2572.

(5) Mullin, J. W. Crystallization: 4th Edition; 2001.

(6) Davis, A. F.; Hadgraft, J. Effect of Supersaturation on Membrane Transport: Hydrocortisone Acetate. Int. J. Pharm. 1991, 76 (1-2), 1-8.

(7) M. A. Pellett, A. F. Davis, J. H. Effect of Supersaturation on Membrane Transport: 2. Piroxicam. Int. J. Pharm. 1994, 111 (1), 1-6.

(8) Santos, P.; Watkinson, A. C.; Hadgraft, J.; Lane, M. E. Enhanced Permeation of Fentanyl from Supersaturated Solutions in a Model Membrane. Int. J. Pharm. 2011, 407 (1-2), 72-77.

(9) Umprakob, U. K.; Awakami, J. K.; Dachi, I. A. Permeation Enhancement of Ketoprofen Using a Supersaturated System with Antinucleant Polymers. Biol. Pharm. Bull. 2005, 28 (9), 1684-1688.

(10) Raina, S. A.; Zhang, G. G. Z.; Alonzo, D. E.; Wu, J.; Zhu, D.; Catron, N. D.; Gao, Y. I.; Taylor, L. S. Enhancements and Limits in Drug Membrane Transport Using Supersaturated Solutions of Poorly Water Soluble Drugs. J. Pharm. Sci. 2014, 103 (9), 2736-2748.

(11) Indulkar, A. S.; Gao, Y.; Raina, S. A.; Taylor, L. S. Exploiting the Phenomenon of Liquid-Liquid Phase Separation for Enhanced and Sustained Membrane Transport of a Poorly Water- Soluble Drug. Mol. Pharm. 2016, 13 (6), 2059-2069.

(12) Ilevbare, G. a.; Taylor, L. S. Liquid-Liquid Phase Separation in Highly Supersaturated Aqueous Solutions of Poorly Water-Soluble Drugs: Implications for Solubility Enhancing Formulations. Cryst. Growth Des. 2013, 13 (4), 1497-1509. 
(13) Brick, M. C.; Palmer, H. J.; Whitesides, T. H. Formation of Colloidal Dispersions of Organic Materials in Aqueous Media by Solvent Shifting. Langmuir 2003, 19 (1), 6367-6380.

(14) Hate, S. S.; Reutzel-Edens, S. M.; Taylor, L. S. Insight into Amorphous Solid Dispersion Performance by Coupled Dissolution and Membrane Mass Transfer Measurements. Mol. Pharm. 2019, $16(1), 448-461$.

(15) Dietschy, J. M.; Sallee, V. L.; Wilson, F. A. Unstirred Water Layers and Absorption across the Intestinal Mucosa. Gastroenterology 1971, 61 (6), 932-934.

(16) Nielsen, P. E.; Avdeef, A. PAMPA — a Drug Absorption in Vitro Model 8. Apparent Filter Porosity and the Unstirred Water Layer. Eur. J. Pharm. Sci. 2004, 22 (1), 33-41.

(17) Korjamo, T.; Heikkinen, A. T.; Mönkkönen, J. Analysis of Unstirred Water Layer in In Vitro Permeability Experiments. J. Pharm. Sci. 2009, 98 (12), 4469-4479.

(18) Stewart, A. M.; Grass, M. E.; Mudie, D. M.; Morgen, M. M.; Friesen, D. T.; Vodak, D. T. Development of a Biorelevant, Material-Sparing Membrane Flux Test for Rapid Screening of Bioavailability-Enhancing Drug Product Formulations. Mol. Pharm. 2017, 14 (6), 2032-2046.

(19) Kesisoglou, F.; Wang, M.; Galipeau, K.; Harmon, P.; Okoh, G. Effect of Amorphous Nanoparticle Size on Bioavailability of Anacetrapib in Dogs. J. Pharm. Sci. 2019, 1-9.

(20) Riethorst, D.; Mols, R.; Duchateau, G.; Tack, J.; Brouwers, J.; Augustijns, P. Characterization of Human Duodenal Fluids in Fasted and Fed State Conditions. J. Pharm. Sci. 2016, 105 (2), 673681.

(21) Carey, M. C.; Small, D. M. Micelle Formation by Bile Salts: Physical-Chemical and Thermodynamic Considerations. JAMA Intern. Med. 1972, 130 (4), 506-527.

(22) Natalini, B.; Sardella, R.; Gioiello, A.; Ianni, F.; Di Michele, A.; Marinozzi, M. Determination of Bile Salt Critical Micellization Concentration on the Road to Drug Discovery. J. Pharm. Biomed. Anal. 2014, 87, 62-81.

(23) Hofmann, A. F.; Mysels, K. J. Bile Salts as Biological Surfactants. Colloids and Surfaces 1987, 30 (1), 145-173.

(24) Mukerjee, P.; Cardinal, J. R. Solubilization as a Method for Studying Self-Association: Solubility of Naphthalene in the Bile Salt Sodium Cholate and the Complex Pattern of Its Aggregation. $J$. Pharm. Sci. 1976, 65 (6), 882-886. 
(25) Mazer, N. A.; Carey, M. C.; Kwasnick, R. F.; Benedek, G. B. Quasielastic Light Scattering Studies of Aqueous Biliary Lipid Systems. Size , Shape, and Thermodynamics of Bile Salt Micelles. Biochemistry 1979, 18 (14), 3064-3075.

(26) Reis, S.; Moutinho, C. G.; Matos, C.; De Castro, B.; Gameiro, P.; Lima, J. L. F. C. Noninvasive Methods to Determine the Critical Micelle Concentration of Some Bile Acid Salts. Anal. Biochem. 2004, 334 (1), 117-126.

(27) Rodas, A.; Hofmann, A. F.; Myselsn, K. J. The Influence of Bile Salt Structure on SelfAssociation in Aqueous Solutions. J. Biol. Chem. 1983, 258 (10), 6362-6370.

(28) Mazer, N. A.; Benedek, G. B.; Carey, M. C. Quasielastic Light-Scattering Studies of Aqueous Biliary Lipid Systems. Mixed Micelle Formationin Bile Salt-Lecithin Solutions. Biochemistry 1980, $19(4), 601-615$.

(29) Claffey, W. J.; Holzbach, R. T. Dimorphism in Bile Salt/Lecithin Mixed Micelles. Biochemistry 1981, 20, 415-418.

(30) Klein, S. The Use of Biorelevant Dissolution Media to Forecast the In Vivo Performance of a Drug. AAPS J. 2010, 12 (3), 397-406.

(31) Jantratid, E.; Janssen, N.; Reppas, C.; Dressman, J. B. Dissolution Media Simulating Conditions in the Proximal Human Gastrointestinal Tract: An Update. Pharm. Res. 2008, 25 (7), 1663-1676.

(32) Lu, J.; Ormes, J. D.; Lowinger, M.; Xu, W.; Mitra, A.; Mann, A. K. P.; Litster, J. D.; Taylor, L. S. Impact of Endogenous Bile Salts on the Thermodynamics of Supersaturated Active Pharmaceutical Ingredient Solutions. Cryst. Growth Des. 2017, 17 (3), 1264-1275.

(33) Li, N.; Mosquera-Giraldo, L. I.; Borca, C. H.; Ormes, J. D.; Lowinger, M.; Higgins, J. D.; Slipchenko, L. V.; Taylor, L. S. A Comparison of the Crystallization Inhibition Properties of Bile Salts. Cryst. Growth Des. 2016, 16 (12), 7286-7300.

(34) Lu, J.; Ormes, J. D.; Lowinger, M.; Mann, A. K. P.; Xu, W.; Litster, J. D.; Taylor, L. S. Maintaining Supersaturation of Active Pharmaceutical Ingredient Solutions with Biologically Relevant Bile Salts. Cryst. Growth Des. 2017, 17 (5), 2782-2791.

Raina, S. A.; Zhang, G. G. Z.; Alonzo, D. E.; Wu, J.; Zhu, D.; Catron, N. D.; Gao, Y.; Taylor, L. S. Impact of Solubilizing Additives on Supersaturation and Membrane Transport of Drugs. Pharm. Res. 2015, 32 (10), 3350-3364. 
(36) Indulkar, A. S.; Gao, Y.; Raina, S. A.; Zhang, G. G. Z.; Taylor, L. S. Crystallization from Supersaturated Solutions: Role of Lecithin and Composite Simulated Intestinal Fluid. Pharm. Res. 2018, 35 (158).

(37) Higuchi; T. Physical Chemical Analysis of Percutaneous Absorption Process from Creams and Ointments. J Soc Cosmet Chem 1960, 11, 85-97.

(38) Indulkar, A. S.; Box, K. J.; Taylor, R.; Ruiz, R.; Taylor, L. S. PH-Dependent Liquid-Liquid Phase Separation of Highly Supersaturated Solutions of Weakly Basic Drugs. Mol. Pharm. 2015, 12, $2365-2377$.

(39) Fang, L. Y.; Wan, J.; Harris, D. Oral Pharmaceutical Compositions in a Solid Dispersion Comprising Preferably Posaconazole and HPMCAS. US2011/0034478A1, 2011.

(40) Hens, B.; Brouwers, J.; Corsetti, M.; Augustijns, P. Supersaturation and Precipitation of Posaconazole Upon Entry in the Upper Small Intestine in Humans. J. Pharm. Sci. 2016, 105 (9), 2677-2684.

(41) Elkhabaz, A.; Sarkar, S.; Simpson, G. J.; Taylor, L. S. Characterization of Phase Transformations for Amorphous Solid Dispersions of a Weakly Basic Drug upon Dissolution in Biorelevant Media. Pharm. Res. 2019, 36 (12), 158.

(42) Raina, S. A.; Alonzo, D. E.; Zhang, G. G. Z.; Gao, Y.; Taylor, L. S. Using Environment-Sensitive Fluorescent Probes to Characterize Liquid-Liquid Phase Separation in Supersaturated Solutions of Poorly Water Soluble Compounds. Pharm. Res. 2015, 32 (11), 3660-3673.

(43) Indulkar, A. S.; Mo, H.; Gao, Y.; Raina, S. A.; Zhang, G. G. Z.; Taylor, L. S. Impact of Micellar Surfactant on Supersaturation and Insight into Solubilization Mechanisms in Supersaturated Solutions of Atazanavir. Pharm. Res. 2017.

(44) Xie, T.; Gao, W.; Taylor, L. S. Impact of Eudragit EPO and Hydroxypropyl Methylcellulose on Drug Release Rate, Supersaturation, Precipitation Outcome and Redissolution Rate of Indomethacin Amorphous Solid Dispersions. Int. J. Pharm. 2017, 531 (1), 313-323.

(45) Ilevbare, G. A.; Liu, H.; Pereira, J.; Edgar, K. J.; Taylor, L. S. Influence of Additives on the Properties of Nanodroplets Formed in Highly Supersaturated Aqueous Solutions of Ritonavir. Mol. Pharm. 2013, 10 (9), 3392-3403.

(46) Kourentas, A.; Vertzoni, M.; Barmpatsalou, V.; Augustijns, P.; Beato, S.; Butler, J.; Holm, R.; Ouwerkerk, N.; Rosenberg, J.; Tajiri, T.; Tannergren, C.; Symillides, M.; Reppas, C. The BioGIT 
System: A Valuable In Vitro Tool to Assess the Impact of Dose and Formulation on Early Exposure to Low Solubility Drugs After Oral Administration. AAPS J. 2018, 20 (71), 1-12.

(47) Augustijns, P.; Wuyts, B.; Hens, B.; Annaert, P.; Butler, J.; Brouwers, J. A Review of Drug Solubility in Human Intestinal Fluids: Implications for the Prediction of Oral Absorption. Eur. J. Pharm. Sci. 2014, 57 (1), 322-332.

(48) Inagaki, I.; Adachi, M.; Ito, H.; Yasuda, M.; Tsurumi, H.; Deguchi, T.; Seishima, M. AtazanavirInduced Urine Crystals Demonstrated by Infrared Spectroscopic Analysis. Urol. Int. 2015, 94 (1), $121-124$.

(49) Daudon, M.; Frochot, V.; Bazin, D.; Jungers, P. Crystalluria Analysis Improves Significantly Etiologic Diagnosis and Therapeutic Monitoring of Nephrolithiasis. Comptes Rendus Chim. 2016, $19(11-12), 1514-1526$.

(50) Takano, R.; Takata, N.; Saito, R.; Furumoto, K.; Higo, S.; Hayashi, Y.; Machida, M.; Aso, Y. Quantitative Analysis of the Effect of Supersaturation on in Vivo Drug Absorption. Mol. Pharm. 2010, 7 (5), 1431-1440.

(51) Megrab, N. A.; Williams, A. C.; Barry, B. W. Oestradiol Permeation through Human Skin and Silastic Membrane: Effects of Propylene Glycol and Supersaturation. J. Control. Release 1995, 36 (3), 277-294.

(52) Wilson, V.; Lou, X.; Osterling, D. J.; Stolarik, D. F.; Jenkins, G.; Gao, W.; Zhang, G. G. Z.; Taylor, L. S. Relationship between Amorphous Solid Dispersion in Vivo Absorption and in Vitro Dissolution: Phase Behavior during Dissolution, Speciation, and Membrane Mass Transport. $J$. Control. Release 2018, 292, 172-182.

(53) Yalkowsky, S. H. Solubility and Solubilization in Aqueous Media; Oxford University Press: New York, 1999.

(54) Poelma, F. G. J.; Breäs, R.; Tukker, J. J.; Crommelin, D. J. A. Intestinal Absorption of Drugs. The Influence of Mixed Micelles on on the Disappearance Kinetics of Drugs from the Small Intestine of the Rat. J. Pharm. Pharmacol. 1991, 43 (5), 317-324.

(55) Katneni, K.; Charman, S. A.; Porter, C. J. H. Permeability Assessment of Poorly Water-Soluble Compounds under Solubilizing Conditions: The Reciprocal Permeability Approach. J. Pharm. Sci. 2006, 95 (10), 2170-2185.

(56) Miller, J. M.; Beig, A.; Krieg, B. J.; Carr, R. A.; Borchardt, T. B.; Amidon, G. E.; Amidon, G. L.; 
Dahan, A. The Solubility-Permeability Interplay: Mechanistic Modeling and Predictive Application of the Impact of Micellar Solubilization on Intestinal Permeation. Mol. Pharm. 2011, $8(5), 1848-1856$.

(57) Berben, P.; Mols, R.; Brouwers, J.; Tack, J.; Augustijns, P. Gastrointestinal Behavior of Itraconazole in Humans - Part 2: The Effect of Intraluminal Dilution on the Performance of a Cyclodextrin-Based Solution. Int. J. Pharm. 2017, 526 (1-2), 235-243.

(58) Shore, P. A.; Brodie, B. B.; Hogben, C. A. M. The Gastric Secretion of Drugs: A PH Partition Hypothesis. J. Pharmacol. Exp. Ther. 1957, 119 (3), 361-369.

Crum, M. F.; Trevaskis, N. L.; Pouton, C. W.; Porter, C. J. H. Transient Supersaturation Supports Drug Absorption from Lipid- Based Formulations for Short Periods of Time, but Ongoing Solubilization Is Required for Longer Absorption Periods. Mol. Pharm. 2017, 14 (2), 394-405.

(60) Palmelund, H.; Madsen, C. M.; Plum, J.; Müllertz, A.; Rades, T. Studying the Propensity of Compounds to Supersaturate: A Practical and Broadly Applicable Approach. J. Pharm. Sci. 2016, 105 (10), 3021-3029.

(61) Blaabjerg, L. I.; Grohganz, H.; Lindenberg, E.; Löbmann, K.; Müllertz, A.; Rades, T. The Influence of Polymers on the Supersaturation Potential of Poor and Good Glass Formers. Pharmaceutics 2018, 10 (4), 1-14.

(62) Gao, P.; Akrami, A.; Alvarez, F.; Hu, J.; Li, L. A. N.; Ma, C.; Surapaneni, S. Characterization and Optimization of AMG 517 Supersaturatable Self-Emulsifying Drug Delivery System (S-SEDDS) for Improved Oral Absorption. J. Pharm. Sci. 2009, 98 (2), 516-528.

(63) Anby, M. U.; Williams, H. D.; McIntosh, M.; Benameur, H.; Edwards, G. A.; Pouton, C. W.; Porter, C. J. H. Lipid Digestion as a Trigger for Supersaturation: Evaluation of the Impact of Supersaturation Stabilization on the in Vitro and in Vivo Performance of Self-Emulsifying Drug Delivery Systems. Mol. Pharm. 2012, 9 (7), 2063-2079.

(64) Suys, E. J. A.; Chalmers, D. K.; Pouton, C. W.; Porter, C. J. H. Polymeric Precipitation Inhibitors Promote Fenofibrate Supersaturation and Enhance Drug Absorption from a Type IV Lipid-Based Formulation. Mol. Pharm. 2018, 15 (6), 2355-2371.

(65) Clarysse, S.; Psachoulias, D.; Brouwers, J.; Tack, J.; Annaert, P.; Duchateau, G.; Reppas, C.; Augustijns, P. Postprandial Changes in Solubilizing Capacity of Human Intestinal Fluids for BCS Class II Drugs. Pharm. Res. 2009, 26 (6), 1456-1466. 
(66) Bevernage, J.; Forier, T.; Brouwers, J.; Tack, J.; Annaert, P.; Augustijns, P. Excipient-Mediated Supersaturation Stabilization in Human Intestinal Fluids. Mol. Pharm. 2011, 8 (2), 564-570.

(67) Hens, B.; Corsetti, M.; Brouwers, J.; Augustijns, P. Gastrointestinal and Systemic Monitoring of Posaconazole in Humans After Fasted and Fed State Administration of a Solid Dispersion. $J$. Pharm. Sci. 2016, 105 (9), 2904-2912.

(68) Rubbens, J.; Brouwers, J.; Tack, J.; Augustijns, P. Gastrointestinal Dissolution, Supersaturation and Precipitation of the Weak Base Indinavir in Healthy Volunteers. Eur. J. Pharm. Biopharm. 2016, 109, 122-129.

(69) Ezzet, F.; Wexler, D.; Courtney, R.; Krishna, G.; Lim, J. Oral Bioavailability of Posaconazole in Fasted Healthy Subjects Dosage Recommendations. Clin. Pharmacokinet. 2005, 44 (2), 211-220.

(70) Söderlind, E.; Karlsson, E.; Carlsson, A.; Kong, R.; Lenz, A.; Lindborg, S.; Sheng, J. J. Simulating Fasted Human Intestinal Fluids: Understanding the Roles of Lecithin and Bile Acids. Mol. Pharm. 2010, 7 (5), 1498-1507.

(71) Hammad, M. A.; Mu, B. W. Increasing Drug Solubility by Means of Bile Salt Phosphatidylcholine-Based Mixed Micelles. Eur. J. Pharm. Biopharm. 1998, 46 (3), 361-367.

(72) Berben, P.; Bauer-brandl, A.; Brandl, M.; Faller, B.; Eide, G.; Jacobsen, A.; Brouwers, J.; Augustijns, P. Drug Permeability Profiling Using Cell-Free Permeation Tools: Overview and Applications. Eur. J. Pharm. Sci. 2018, 119, 219-233.

(73) Persson, E. M.; Gustafsson, A.; Carlsson, A. S.; Nilsson, R. G.; Knutson, L.; Forsell, P.; Hanisch, G.; Lennerna, H. The Effects of Food on the Dissolution of Poorly Soluble Drugs in Human and in Model Small Intestinal Fluids. Pharm. Res. 2005, 22 (12), 2141-2151.

(74) Heikkilä, T.; Karjalainen, M.; Ojala, K.; Partola, K.; Lammert, F.; Augustijns, P.; Urtti, A.; Yliperttula, M.; Peltonen, L.; Hirvonen, J. Equilibrium Drug Solubility Measurements in 96-Well Plates Reveal Similar Drug Solubilities in Phosphate Buffer PH 6.8 and Human Intestinal Fluid. Int. J. Pharm. 2011, 405 (1-2), 132-136.

(75) Clarysse, S.; Tack, J.; Lammert, F.; Duchateau, G.; Reppas, C.; Augustijns, P. Postprandial Evolution in Composition and Characteristics of Human Duodenal Fluids in Different Nutritional States. J. Pharm. Sci. 2009, 98 (3), 1177-1192. 
Table of Contents Graphic

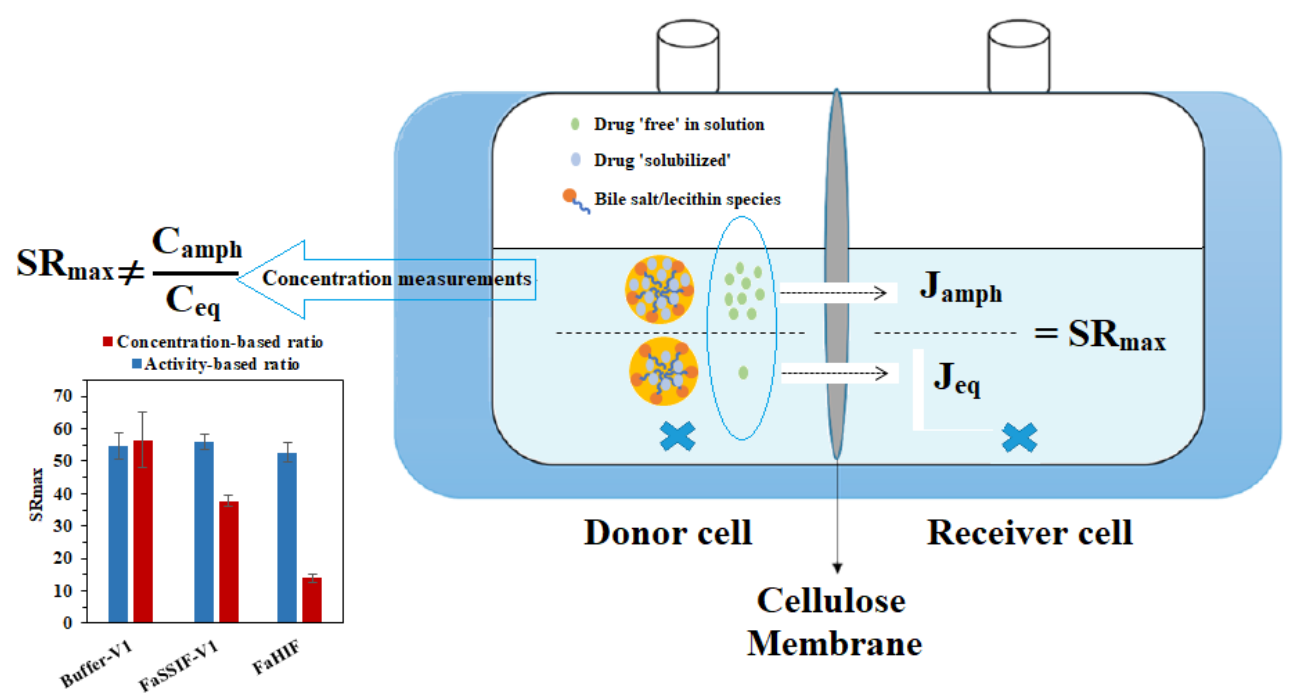

